\title{
Strong scaling of general-purpose molecular dynamics simulations on GPUs
}

\author{
Jens Glaser ${ }^{\mathrm{a}}$, Trung Dac Nguyen ${ }^{\mathrm{c}}$, Joshua A. Anderson ${ }^{\mathrm{a}}$, Pak Lui ${ }^{\mathrm{d}}$, Filippo \\ Spiga $^{\mathrm{e}}$, Jaime A. Millan ${ }^{\mathrm{b}}$, David C. Morse ${ }^{\mathrm{f}}$, Sharon C. Glotzer ${ }^{\mathrm{a}, \mathrm{b}, *}$ \\ ${ }^{a}$ Department of Chemical Engineering, 2800 Plymouth Rd, University of Michigan, Ann Arbor, \\ MI 48109, USA \\ ${ }^{b}$ Department of Materials Science and Engineering, 2300 Hayward St, University of Michigan, \\ Ann Arbor, MI 48109, USA \\ ${ }^{c}$ National Center for Computational Sciences, Oak Ridge National Laboratory, TN 37831, USA \\ ${ }^{d}$ Mellanox Technologies, Inc., 350 Oakmead Parkway, Sunnyvale, CA 94085, USA \\ ${ }^{e}$ High Performance Computing Service, University of Cambridge, 17 Mill Lane, Cambridge, CB2 \\ $1 R X, U K$ \\ ${ }^{f}$ Department of Chemical Engineering and Materials Science, 421 Washington Street SE, \\ Minneapolis, MN 55455, USA
}

\begin{abstract}
We describe a highly optimized implementation of MPI domain decomposition in a GPU-enabled, general-purpose molecular dynamics code, HOOMD-blue (Anderson and Glotzer, arXiv:1308.5587). Our approach is inspired by a traditional CPU-based code, LAMMPS (Plimpton, J. Comp. Phys. 117, 1995), but is implemented within a code that was designed for execution on GPUs from the start (Anderson et al., J. Comp. Phys. 227, 2008). The software supports short-ranged pair force and bond force fields and achieves optimal GPU performance using an autotuning algorithm. We are able to demonstrate equivalent or superior scaling on up to 3,375 GPUs in Lennard-Jones and dissipative particle dynamics (DPD) simulations of up to 108 million particles. GPUDirect RDMA capabilities in recent
\end{abstract}

\footnotetext{
${ }^{*}$ Corresponding author

Email address: sglotzer@umich.edu (Sharon C. Glotzer)
}

Preprint submitted to Computer Physics Communications

December 10, 2014 
GPU generations provide better performance in full double precision calculations. For a representative polymer physics application, HOOMD-blue 1.0 provides an effective GPU vs. CPU node speed-up of $12.5 \times$.

Keywords: Multi-GPU, Molecular Dynamics, MPI/CUDA, strong scaling, weak scaling domain decomposition, LAMMPS

PACS: 02.70.Ns, 65.20.De, 61.25.H-

\section{Introduction}

Graphics processing units (GPUs), massively parallel processors with thousands of compute cores, represent a disruptive technology shift in simulation hardware. Molecular dynamics (MD) simulations once requiring tens or hundreds of CPU cores are now routinely performed on the researcher's desktop workstation using only a single GPU. All major MD software packages now take advantage of single-GPU acceleration, and some of them offer multi-GPU capabilities. However, as many-GPU clusters and petascale class supercomputers such as Ti$\tan (18,688$ GPUs) and Blue Waters (4,200 GPUs) are becoming a mainstay of scientific computing, making efficient use of these powerful resources is key to productivity.

HOOMD-blue, which was the first general-purpose molecular dynamics code written exclusively for NVIDIA GPUs [1], is extended to run efficiently on thousands of GPUs in parallel. Today, many, if not all major molecular dynamics codes support GPU acceleration, including AMBER [2], LAMMPS [3, 4, 5, 6] GROMACS [7], NAMD [8, 9], CHARMM [10], DL_POLY [11], ACEMD [12], Desmond [13], Espresso [14], and Folding@Home [10]. Since these codes were designed with CPUs in mind, they take advantage of GPUs at different levels of 
efficiency. In most of these codes, only the dominant compute-intensive part of the algorithm has been ported to the GPU. The advantage of such an approach is that it offers significant speed-up vs. a single CPU core without the need to rewrite a legacy application. However, it also means that the code does not take maximum advantage of the GPU if the particle data structures are not GPU-optimized and data needs to be copied back and forth between the CPU and the GPU. Codes designed exclusively for the GPU include Fen-Zi [15] or HALMD [16], which implement only a limited feature set. HOOMD-blue is unique among all these codes. It uses completely device-resident data structures, all simulation work is performed on the GPU so that the CPU merely acts as a driver for the GPU, and it offers a rich feature set for general-purpose particle-based simulations [17].

The first HOOMD releases up to 0.11 .3 are highly-tuned for single-GPU performance and do not support multi-GPU runs. Reducing latency is one of the biggest challenges in developing a code scalable to many GPUs. Data transferred between GPUs moves over the PClexpress bus (PCIe), whose bandwidth (up to $16 \mathrm{~GB} / \mathrm{s}$ ) and latency (several $\mu \mathrm{s}$ ) is much slower than on-board GPU memory (250GB/s, $100 \mathrm{~ns})$. Communicating over PCle adds latency that is not present in single-GPU runs. In the strong scaling limit of increasing the number of GPUs $P$ at constant number $N$ of particles, the work $N / P$ performed by each GPU decreases to the point where it is too small to fully utilize the device. Finally, whenever possible, communication should be overlapped with computation to mask latency.

In developing HOOMD-blue 1.0 with MPI spatial domain decomposition, we addressed these challenges and here we demonstrate scaling on over 3,000 GPUs. We show that strong scaling speed-ups in excess of 50x are attainable on the Titan 
supercomputer, and weak scaling holds over three orders of magnitude in system size. We compare HOOMD-blue to three other implementations of molecular dynamics (LAMMPS-GPU [5], LAMMPS USER-MESO [6] and LAMMPSKokkos [18]) on GPUs and show significant advances in scaling over these implementations. Moreover, we examine the efficiency of CUDA-aware MPI and demonstrate superior performance of GPUDirect RDMA [19], in combination with a high-performance MPI implementation, MVAPICH2 2.1 [20], for some use cases.

The remainder of the paper is organized as follows. In Sec. 2, we give an overview of the research areas within soft condensed matter physics in which we expect the GPU-based domain decomposition approach to be helpful, followed by a description of the capabilities of the present HOOMD-blue version in Sec. 3 . Section 4 presents a detailed description of the new communication algorithm. In Sec. 5 we further discuss how we optimized the code for strong scaling on many GPUs. We show results for weak and strong scaling of a Lennard-Jones (LJ) benchmark in Sec. 6. We show scaling data for a polymer brush system with various short-ranged force fields in Sec. 7. Subsequently we discuss the efficiency of GPUDirect RDMA for strong scaling (Sec. 8). In Sec. 9, we discuss a different benchmark system, DPD. We conclude (Sec. 10) with an outlook on future enabling technologies for GPU-based MD.

\section{Scientific applications}

HOOMD-blue's modular and compartmentalized, yet easy-to-use design allows customizability and flexibility for a wide range of applications, as exemplified by the over 80 published works that use this software to date [21]. Previously, 
the software has been used for single-GPU simulations, and for weakly coupled parallel tempering simulations. Here, we report on how its features have been enabled with MPI spatial domain decomposition, and give examples of soft condensed matter research where such functionality may be useful, such as polymer systems and complex fluids.

Simulations of coarse-grained, bulk polymeric liquids are used for detailed investigations into the thermodynamic properties of model materials. In those studies, long polymer chains are required to reduce discretization effects. Large simulation unit cells are necessary because of the scale separation between a monomer and the polymer coil, and to simulate many such coils (radii of gyration). Typically, bulk polymer liquids or melts have a homogeneous monomer density and often these systems are effectively incompressible. Such simulations are particularly amenable to the domain decomposition approach [22, 23]. Similarly suited are, e.g., simulations of polymer solutions [24], micelle aggregation [25], polymer brushes [26], wetting/dewetting phenomena [27, 28, 29], and glassy polymers [30]. Heterogeneous systems, such as micelles in implicit solvent using DPD or Brownian dynamics, are susceptible to load imbalances [31] and may map less well onto the domain decomposition approach.

More complex simulation scenarios include anisotropic particles, polymer nanocomposites such as tethered nanoparticles [32], colloidal crystals [33], structure formation from isotropic pair potentials [34], actively driven or self-propelled particles [35], and biological applications [36, 37, 38]. The emergent structures of interest, such as a crystal unit cell or dynamical swarms and clusters, are often much larger than the size of the constituent building blocks or agents, and many such units or features need to be simulated, requiring large-scale simulations. Be- 
cause of the complexity of the interactions, these simulations tend to be more compute-intensive and should scale well.

\section{Characterization of HOOMD-blue}

HOOMD-blue is a general-purpose code, though it primarily targets softmatter and coarse-grained simulations. Many other specialized MD codes are available for all-atom models. As a a general-purpose MD code, HOOMD-blue is highly customizable and comes with many features, most of which have been enabled in MPI mode. All of HOOMD-blue's capabilities are available both on the GPU and the CPU, including MPI domain decomposition, and the CPU can be used when no GPU is available or for testing purposes. Here we briefly describe the features that have been ported to the MPI version. Existing functionality from previous versions has been fully preserved in single GPU/core runs. Utilizing the MPI functionality does not require any changes to existing job scripts.

HOOMD-blue is driven by Python simulation scripts. In multi-GPU/core simulations, multiple Python interpreters on different MPI ranks process the same input script. The main simulation engine is written in CUDA and $\mathrm{C}++$. In HOOMD-blue 1.0, full double precision is available per compile-time option. HOOMD-blue version 1.0 is optimized for Kepler generation GPUs and also runs on the previous generation, Fermi. HOOMD-blue is optimized for each new generation of GPUs as it becomes available. Auto-tuning is used to automatically optimize GPU kernel execution parameters at runtime (see also Sec. 5.2).

We needed to update a majority of the classes in HOOMD-blue to support MPI, including the file I/O classes, integrators, pair and bond potentials, and analyzer classes. File I/O-classes include the initialization and saving of checkpoints 
through HOOMD-blue's XML file format, and output of simulation trajectories, using CHARMM's DCD file format. Integrators include NVE (constant energy), NVT (isothermal- isochoric), NPH (isotenthalpic-isobaric) and NPT (isothermalisobaric) ensembles, which implement the Velocity-Verlet or the symplectic MartynaTobias-Klein update equations [39, 40] for maximum ensemble stability. Brownian (or Langevin) dynamics and DPD [41] are supported as well. Supported pair potentials include LJ, DPD, Gaussian, Morse, Yukawa and tabulated potentials. For molecular simulations (with covalent bonds), bond potentials, i.e. harmonic, FENE and tabulated bonds, and also harmonic and tabulated angle and dihedral potentials, were updated. Different forms of external potentials, such as constant forces and periodically modulated forces, are now supported as well.

\section{Implementation of the communication algorithm}

References [1] and [42] describe the general data layout and GPU kernel implementation. We focus on a description of the communication capabilities in this work.

\subsection{General strategy}

We implement domain decomposition in HOOMD-blue within the existing CUDA/C++/Python framework. The single-GPU version of HOOMD-blue does not transfer any significant data between the host and the device during simulation. In the multi-GPU version, data transfer occurs at instants of communication on every time step. Communication is an optional feature in HOOMD-blue, and does not compromise the performance of the highly optimized single-GPU code path. HOOMD-blue runs the single-GPU code path when executed on one MPI rank, which results in apparently low scaling efficiency when executing on two 
MPI ranks, since this additionally activates the communication routines. However, the communication routines are designed to perform well on many, up to several thousands, of GPUs. Communication occurs only along the necessary dimensions, i.e. the dimension along which the domain is split into sub-domains, and the usual minimum image convention for periodic boundaries is employed along the other dimensions. The domains are decomposed according to a minimum interface-area rule.

HOOMD-blue can optionally take advantage of CUDA-aware MPI libraries (by enabling the compilation setting ENABLE_MPI_CUDA=ON). At the time of writing, this includes MVAPICH2 (version 1.9 and later), and OpenMPI (version 1.7 and later). We exploit these features to optimize the communication of ghost particles because that is the largest communication bottleneck. Given a GPU device memory address, the MPI library can then implement any hardware(or software) based optimizations to accelerate data transfer between the GPU and a second GPU or the network interface card (NIC), including RDMA-accelerated peer-to-peer copying and GPUDirect RDMA [43].

\subsection{Domain decomposition}

Domain decomposition and inter-node communication in HOOMD-blue 1.0 follow the same basic approach as in the LAMMPS [3] molecular dynamics simulator: a spatial domain decomposition on a regular one-, two or three-dimensional processor grid is employed, and boundary (or so called 'ghost') particles are communicated to compute the short-ranged pair forces ${ }^{1}$ (Fig. 1). Particle migration

\footnotetext{
${ }^{1}$ The current version of HOOMD-blue supports only short-ranged molecular dynamics force fields in multi-GPU mode.
} 
transfers ownership of particles between neighboring domains and occurs with every rebuild of the neighbor list. Neighbor list builds occur when particles move more than half of the buffer length to guarantee correct force computations. The sum of cut-off and buffer length sets the width of the ghost layer, which becomes invalid when the neighbor list is rebuilt. Between neighbor list builds, particles may move beyond the boundaries of the local domain without migration. Positions (and, depending on the force field, velocities or orientations) of ghost particles are updated every time step, which requires communication.

\subsection{GPU-based communication algorithm}

The core of HOOMD-blue's new MPI capabilities is the communication algorithm, which features a number of optimizations to reduce unnecessary devicehost data transfers, and in particular allows sending/receiving particle data directly to/from the device-side data structures. The basic particle packing and unpacking routines are fully implemented on the GPU.

\subsubsection{Particle migration}

Particle data is stored in a structure of dense arrays for optimal execution of communication kernels on the GPU. When particles are migrated, the particle data is reordered by first removing particles that leave the domain, which creates holes in the arrays. These are removed using a stream compaction based on the scan primitive [44]. Subsequently new particles are added at the end of the arrays. Even though the simultaneous reordering of the GPU arrays for position, velocity, acceleration etc. is a relatively expensive operation, it is performed only infrequently, typically every 5-20 time steps when the neighbor list needs to be rebuilt. 
Figure 1: (color online) Domain decomposition with ghost particles and periodic boundary conditions. Shaded regions at the domain boundaries correspond to ghost particle layers. Short-ranged pair forces require a neighbor cut-off distance $r_{\text {cut }}$ and a buffer length $r_{\text {buff }}$, and their sum defines the ghost layer width. 
In LAMMPS, the exchange with the neighboring domains is reduced to six communication calls, including exchanges along the east-west, north-south, and up-down directions, which is the minimum required in three spatial dimensions [3]. The approach bundles several smaller messages into fewer large messages, to increase communication bandwidth utilization. For GPU implementation, however, this is a disadvantage, since it introduces data-dependency between the exchanges, as the receive buffers for particles from, say, an eastern neighbor need to be scanned for particles that must be forwarded in the next exchange in the north direction. In particular, an extra device-host copy and a kernel call between every exchange is incurred. which increases latency. We determine that simultaneously communicating with all 26 neighbors using non-blocking MPI calls performs better in all cases. The MPI library can optimally pipeline between those transfers.

To limit data transferred over PCIe, we pack the buffer of particles to send on the GPU in a device-gather operation. The data, which represents only a small subset of particles in a thin slab near the boundary of every domain, is copied to the host and the host buffer is then passed to the MPI library. Receiving particles works analogously. We generally use mapped and pinned memory for host buffers to obtain higher bandwidth and compute-data transfer overlap. In some circumstances, however, non-mapped (explicitly staged) memory is faster. We evaluate the performance of both modes at run time and select the fastest using auto-tuning (see Sec. 5.2).

\subsubsection{Ghost particle exchange}

Ghost particles are exchanged after particle migration. They are appended to the particle data arrays and remain valid until the next exchange. A ghost particle can be simultaneously a neighbor in up to three domains - one 'face' neighbor, 
one 'edge' neighbor and one 'corner' neighbor. The same particle needs to be copied into up to three output buffers. While this represents a straight-forward operation to do on the CPU, efficient parallel replication of ghost particles on the GPU requires a more elaborate implementation. We implement particle replication as summarized in Fig. 2. First, a set of flags (a bitfield) is initialized for every particle that indicate which neighboring directions the ghost particle is supposed to be sent to (such as: north-west). Second, the algorithm counts the number of neighboring boxes the particle is replicated to and writes pairs of particle index and destination rank for every replication into an intermediate buffer. Multiple destination ranks can map to the same particle index, so multiple output elements are generated per input element. To implement such an operation efficiently using an atomics-free algorithm, i.e. an algorithm which does not rely on random ordering of parallel read/write accesses to the same location, we turn to the loadbalancing search or 'expand' algorithm within the ModernGPU framework [44]. We adapt this routine to perform both the expansion of particle indices and the determination of neighbor ranks in a single kernel call. Subsequently, output elements are sorted by destination rank (using the MergeSort algorithm [44]), and the begin and end indices for each destination rank are found using vectorized binary search and mark the boundaries of the send buffer. These optimizations resulted in significant speed-up of the ghost exchange over an implementation that involves many different kernel calls (e.g. using Thrust [45]).

\subsubsection{Overlapping communication and computation and other optimizations}

We evaluated two possible strategies for overlapping communication and computation, (i) splitting the force computation into particles with local- and nonlocal neighbors, and (ii) overlapping collective MPI calls with computation. 


\section{Ghost particle exchange:}

1. Mark ghost atoms for sending

2. In one kernel, determine destination ranks for every ghost particle and write out (particle index, destination rank) pairs into a ghost particle list where a particle index can occur multiple times, using the loadbalancing 'expand' operation

3. sort list by destination rank and determine start and end indices of every rank

4. gather particle positions (velocities, ..) of ghost atoms according to ghost particle list into send buffer

Figure 2: Ghost particle exchange optimized using load-balancing search [44]. 
In the first approach, the force computation for every local particle is split into the forces due to local particles, and forces due to ghost particles. The computation of forces due to interactions with local particles can be overlapped with the communication of ghost particles, after the completion of which the forces due to ghost particles are computed in a separate kernel call. However, because this approach requires two (instead of one) invocations of the force kernel, it incurs launch overhead for the extra kernel. We tested this approach and find that is more expensive than a non-overlapping, single-kernel approach.

In the second approach, forces are always computed using the current particle neighbors, before the results of the neighbor list distance check are known. Since the distance check requires a global synchronization (an MPI collective), this synchronization can now be performed simultaneously with the force computation. When the distance check returns positive, the precomputed force must be discarded, particles migrated, and then correct forces computed. For typical benchmarks where particle migration occurs every 5-20 time steps, the proposed scheme results in better performance. Because theoretially, situations can occur in which performance is adversely affected, in particular for short neighbor list rebuild intervals, the performance difference is measured at run-time and compute/communication overlap is auto-tuned.

A further optimization of the communication pattern is to group global synchronization calls and neighborhood communication into different phases of the time step. Examples for global synchronization include broadcast of parameters during NVT integration, or MPI reduction calls for computation of thermodynamic properties such as kinetic energy, virial etc. Call-back slots are provided in the communication algorithm for different HOOMD classes to opt-in to the op- 
timized execution pattern, to the extent this is possible given data-dependencies. The grouping improves performance because it mitigates the effect of load imbalances, and global synchronization occurs only at one instant during the time step, instead of being interleaved with computation or neighborhood communication.

Because our communication algorithm does not make use of atomic operations, it is fully deterministic, i.e. the simulation results are bit-wise reproducible (e.g. for debugging). However, HOOMD-blue's cell list algorithm uses atomic operations, and overall execution is therefore non-deterministic. We stress that non-determinism arises here as a side-effect of parallel execution (that can sometimes be avoided), due to the non-associativity of floating point operations at finite numerical precision. Since MD integrators are affected by numerical precision in the same way, lack of determinism does not imply degraded stability in a thermodynamic sense.

As a minor optimization, we map the Cartesian rank index to a logical MPI rank, so as to group spatial domains of the decomposition together that are executed on the same physical node. For the neighborhood exchanges this reduces inter-node communication, which is more expensive than intra-node communication.

\subsubsection{Bonded forces}

In HOOMD-blue 1.0, two-particle bonds, three-particle angles and four-particle dihedrals and impropers are treated as instantiations of a generic $\mathrm{C}++$ class template, BondedGroup, to avoid code duplication for different types of particle groups with small numbers of members. For simplicity, we refer to a 'bonded group' as a bond in what follows, implying that the description applies to angles, dihedrals and impropers in an analogous way. A bond is simply an unordered pair of particle 
IDs, together with a bond type number.

Bond data structures are dynamic, and bonds can be added or removed during the simulation using the python scripting interface. Also, in MPI simulations, bonds are stored locally and are migrated between domains together with the particles, which ensures $O(N / P)$ memory and compute scaling for bonds. We developed an efficient communication algorithm for bonded particles. Remarkably, our algorithm does not explicitly require specifying a maximum bond-length, which would otherwise be used to simplify the treatment of ghost particles, because bond neighbors would be treated in the same way as non-bonded neighbors by simply widening the ghost layer to the maximum bond length. For long or very floppy bonds, this would result in the communication of too many non-bonded neighbors.

In our algorithm (summarized in Fig. 3), bond migration occurs directly before particle migration, but after local particles have been identified that leave the domain. The difference between the two steps, besides the difference in the data objects that are communicated, is that particles are uniquely local to a single domain but bonds can span two domains. If a bond is split because one of its members migrates to a neighboring domain, the bond is stored simultaneously in both processors' data structures, and a bond is only removed from a processor's storage if none of its particle members are local anymore. At the time the system is initialized, bonds are distributed onto processors.

The issue of correct force computation is a little more involved, because it is necessary to exchange ghost particles with a neighboring domain if they share a split bond. However, the sending processor does not know a-priori where to send the ghost particles, since the bond can span any of the processor boundaries. In particular, the fact that a bond member is in the proximity of a boundary does 


\section{Bond migration:}

1. (compute) Mark particles that leave the local domain for sending (also used for particle migration)

2. (communicate) For all particles leaving the domain that are members of incomplete bonds, update neighbors with destination processor rank

3. (communicate) For all particles leaving the domain and members of bonds, copy bonds to destination rank

4. (compute) Prune bonds that have no local members

\section{Bonded ghost particle exchange:}

6. (communicate) Using the table constructed or updated in step 2, exchange ghost particles with neighbors

Figure 3: Communication of bonded groups (bonds, angles and dihedrals). 
not imply that the bond actually spans that boundary. To account for this issue, it is necessary to keep track of the processor ranks that share the bond, using a data structure to store all processor ID's that own any member particles. This data structure is initialized at the beginning of the simulation using a broadcast operation between neighbors. It is then updated with every particle migration, by exchanging information with the processors that own other bond members. Hence, bond migration consists of two communication steps, where in the first step we propagate the IDs of particles that leave their domain to the neighboring processors, allowing them to update their rank tables, and in the second step we actually migrate (or replicate) the bonds.

Using current information about bond member ownership, it is straightforward to identify the neighbors with which ghost particles have to be exchanged in order to compute the bond force. Particles are marked for sending in a specific direction, e.g. up-north-west, based on that information. Subsequently, these ghost particles are treated the same way as nonbonded ghost particles, see Sec. 4.3.2.

Communication only occurs between neighboring domains, so bonds cannot span more than two domains, and because of the periodic boundary conditions bond lengths are limited to half the local domain size.

\section{Optimizations for strong scaling}

Ideal $N / P$ scaling of a multi-processor simulation of $N$ particles on $P$ GPUs not only requires low-latency communication routines, but also that all parts of the computation exhibit, linear $N / P$ scaling. As the workload $N / P$ on a single GPU decreases with increasing numbers of processors at constant total system size $N$ in the strong scaling limit, optimal kernel launch parameters for the pair potential 


\section{for all particle $i$ in groups of $w$ parallel threads do:}

1. Look up cell neighbors of particle $i$

2. Iterate over cell neighbors $k$ with a stride of $w$ (block thread $j<w$ processes neighbor with offset $j$ )

3. Set is_neighbor $=1$ if particle is a neighbor $\left(r<r_{\text {cut }}\right)$

4. $o=j$ th element of $w$-wide exclusive prefix sum over has_neighbor

5. $\mathrm{n}=$ result of reduction $(j+1$ th element of prefix sum $)$

6. If is_neighbor $>0$, write neighbor index $k$ into neighbor list at position $n_{-}$tot $+\mathrm{n}$

7. $\mathrm{n} \_$tot $+=\mathrm{n}$

8. Store $n_{-}$tot

Figure 4: Neighbor list algorithm using $w$ cooperative threads per particle.

kernels change. These low-level parameters define how the Single-InstructionMultiple-Threads (SIMT) model maps onto the GPU workload. In previous versions of HOOMD-blue one particle was mapped onto one GPU thread, which ceases to be efficient at low numbers of particles $(N / P \lesssim 20,000)$ because the GPU is underutilized. Therefore, we now assign multiple threads to a particle [46].

\subsection{Force computation with a cooperative thread array}

Instead of processing all particle neighbors in a single loop per thread, we iterate over nonbonded particle neighbors using an array of $w$ cooperative threads. The parameter $w$ must be less than or equal to 32 in the current GPU generation 
because this is the number of threads that are executed in a warp and can communicate with the warp shuffle instruction. In HOOMD-blue 1.0, both the neighbor list and the force computation kernels therefore take $w$ as an additional input parameter, which controls the size of the block. Here, $w=2^{m}$ is a power of two, and if $m<5$ a single warp is split into multiple segments.

The pseudo-code for the neighbor list algorithm is shown in Fig. 4. The algorithm runs in parallel in blocks of width $w$, and loops through the cell neighbors of every particle in a strided fashion (with offset equal to the offset of the thread in the block), to compute the neighbors that are within the cut-off distance. The final result is obtained by compacting all neighbor particle indices into one (output) row of the neighbor list, using intra-warp stream compaction. Threads of a warp segment can communicate with each other via the fast __shfl intrinsic (on Kepler) or via shared memory (on Fermi).

Similarly, for the force computation the work for particle $i$ is parallelized over its neighbors, processing $w$ elements of its neighbor list in parallel, again in a strided fashion. Each of these threads contributes to the total force on particle $i$. After the partial forces have been computed by the $w$ threads, an intra-warp floating point reduction is applied to total up the particle force, which then is written to global memory.

\subsection{Autotuner}

Both the neighbor list and the force computation kernels now depend on $w$, in addition to the thread block size, which is another parameter in the SIMT execution model equal to the number of threads that share the same thread-local memory. However, the block size is only relevant for performance. Because we now have two parameters to tune, it is no longer practical to manually select the 
optimal parameters out of $160=32 \times 5$ (block size $b=n \times 32 b \leq 1024$ [47], and $w=2^{m}$ with $m \leq 5$ ) possible combinations. Moreover, the optimal parameters may change in a single simulation, as a function of density or the pair potential parameters.

Our solution is to implement an autotuner, which measures kernel execution time for every parameter. The tuner sweeps through the possible parameter combinations during the simulation, and compares the median run time from five different kernel executions with the same parameters among different parameter sets. After having found the value that results in the shortest execution time, this optimal value is fixed for a predefine number of MD time steps. The tuning typically takes between 15,000 and 20,000 steps to complete, which in most cases represents a small fraction of the overall number of steps. Periodically, the tuner scans through the parameters to re-adjust the optimal parameter setting.

\subsection{Scaling of compute kernels vs. communication}

We analyze the performance of individual parts of the simulation in Fig. 5 (upper panel). The main plot shows the time spent in different subroutines in a multiGPU simulation as a function of the number of GPUs. As is evident from that plot, the computationally most expensive subroutines - the neighbor list construction and the pair force kernels - scale essentially inversely linear with the number of GPUs, $t_{\text {step }} \sim N / P(N=64,000=$ const. $)$, down to (at least) 4,000 particles per GPU, as a result of the optimizations for strong scaling described in Sec. 5.1 and auto-tuning. However, the time spent in communication (green curve), which occurs every time step, is almost independent of the number of particles per GPU, imposing constant latency every simulation time step. Particularly, we infer from the cross-over of the two curves between $P=4 \ldots 8$, that scaling must break 
Figure 5: (color online) Time $t_{\text {step }}=t_{\text {loop }} / n_{\text {steps }}$ in individual parts of the algorithm for a $N=$ 64, 000 Lennard-Jones liquid benchmark on $P=1 \ldots 16$ K20X GPUs, showing contribution of pair force, neighbor list and dominant communication (ghost update) routines. Inset: Contribution of the migration, ghost exchange and update phases to overall time spent in communication. 
down at fewer than $\sim 10,000$ particles per GPU, where communication becomes the limiting step.

We further detail the contribution of the three phases of communication (see Sec. 4.3) to the average time per step in the inset of Fig. 5, and while all contributions depend only weakly (if at all) on the number of particles per GPU in the regime studied here, the dominant amount of time is spent inside the ghost update occurring every time step. It is therefore this part of the communication that we heavily optimize, through overlap with global synchronization calls (Sec. 4.3.3) and exploitation of CUDA-aware MPI calls (Sec. 4.1 and below).

\section{Scaling performance}

\subsection{Weak scaling}

A first requirement for a scaling code is its capability of handling large simulation workloads, which requires $O(N / P)$ memory scaling. We perform a weakscaling benchmark of a LJ fluid on the ORNL Titan Cray XK7, keeping the number of particles per GPU constant at $N / P=32,000$. The reasons for the choice of the rather simple LJ benchmark, instead of benchmarking e.g. a molecular system, are that it is (i) computationally inexpensive, and (ii) a standard benchmark for MD. Because of (i), any performance issues with the communication algorithm will be exposed, and because of (ii) we can compare, e.g., to the LAMMPS software package with its similar feature set. We perform the benchmark in the constant temperature (NVT) ensemble with a Nosé-Hoover thermostat, albeit one that is not completely symplectic ${ }^{2}$, and which performs one collective synchro-

\footnotetext{
${ }^{2} \mathrm{~A}$ symplectic MTK integrator for NVT was introduced in HOOMD-blue 1.0.1 for improved stability over the the original Nosé-Hoover NVT thermostat, which is deprecated in that version.
} 
nization for kinetic energy summation every time step.

In particular, we simulate a fluid of up to $N=108,000,000$ particles on 3,375 GPUs. The system has been equilibrated from a random initial configuration over 100, 000 time steps, at packing fraction 0.2 , and with a time step of $\delta t=0.002$ (all quantities in self-consistent or LJ units). The particles interact via the 12-6 LJ potential with $\sigma=1.0$, cut off at $3.0 \sigma$. The interaction parameter is $\epsilon=1.0$. The buffer length $r_{\text {buff }}$ is determined as the value that yields the highest number of time steps per second in a preliminary tuning run, and the distance check criterion is applied with the minimum frequency at which no dangerous builds are observed. Subsequent to an auto-tuner warm-up period of 20,000 time steps, the simulation performance we report is averaged over 10,000 steps.

The corresponding LAMMPS-GPU benchmark was performed on the same machine, starting from the same equilibrated initial configuration, as well as the same simulation parameters, but the optimal configuration was determined to have a fixed buffer size (skin length) of 0.4 , and neighbor lists were rebuilt every 5 time steps. LAMMPS-GPU performance benefits from using more than one CPU core to drive the single GPU per node. Therefore, the number of MPI ranks per node was varied between 1 and 16. For every number of nodes, the fastest value of the number of processes per node was selected. To facilitate the initialization of such large systems, the cubic simulation box configuration was replicated along three spatial dimensions, using LAMMPS' replicate command, or HOOMD-blue's system.replicate() method.

Figure 6 shows the number of time steps per second as a function of the

The symplectic integrator requires multiple collective synchronization calls per time step. 


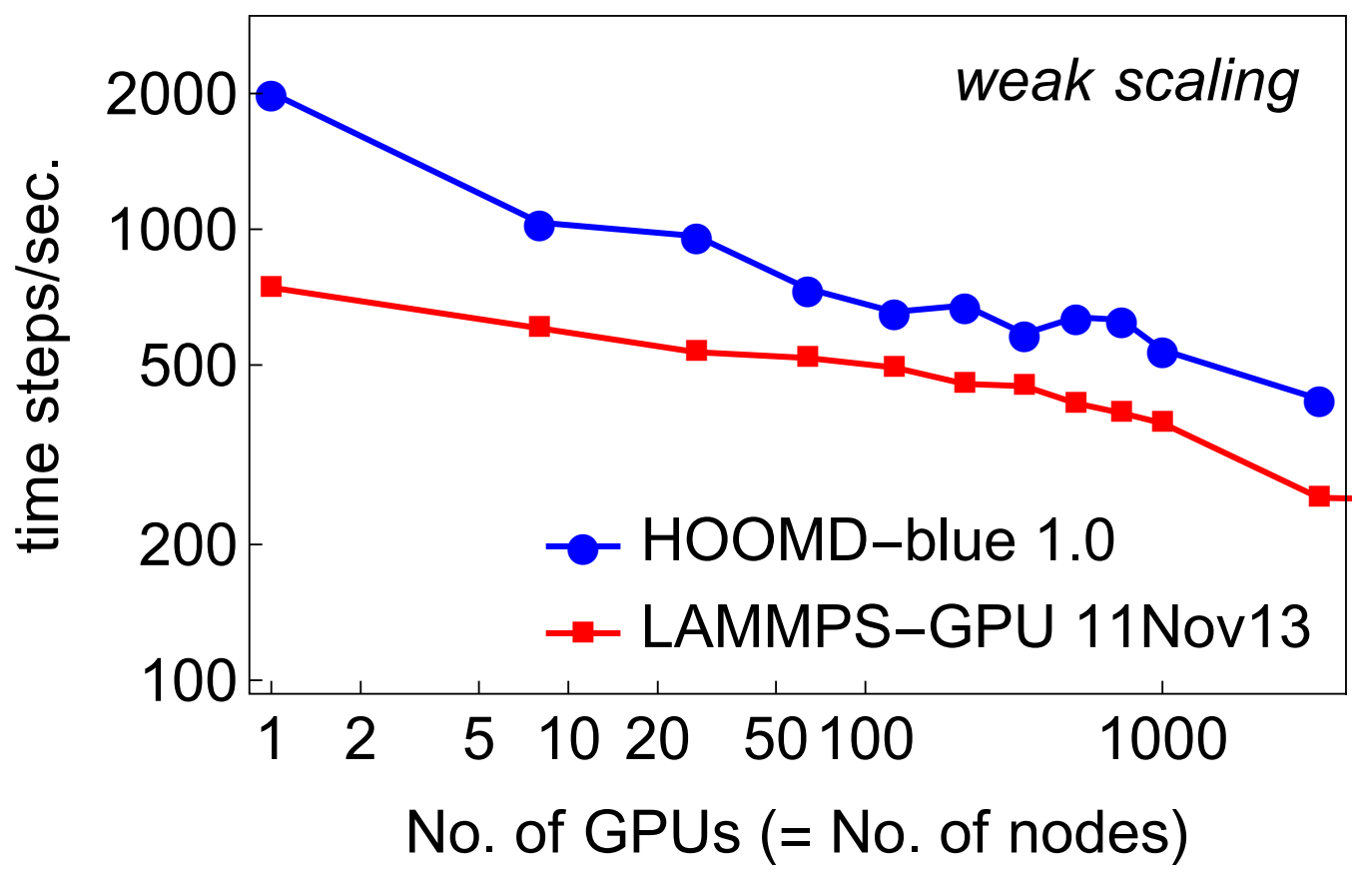

Figure 6: (color online) Weak scaling performance of HOOMD-blue (solid circles) and LAMMPS-GPU (solid squares) on Titan, in number of time steps per second vs. number of GPUs, for a constant number of particles per GPU $N / P=32,000$, for $1 \ldots 3,375$ GPUs. 
number of GPUs used in the weak scaling simulation, for HOOMD-blue and LAMMPS-GPU. Data is shown for up to the maximum total system size we succeeded to initialize on Titan using HOOMD-blue. Ideal weak scaling corresponds to constant performance as a function of the number of GPUs. As expected, the largest drop in performance for HOOMD-blue occurs when the number of GPUs increases from one to $\sim 8$ GPUs, which corresponds to switching from no communication to a fully three-dimensional $(2 \times 2 \times 2)$ domain decomposition. The further decrease in performance of only about $50 \%$ over a range of over 3,000 GPUs is likely caused by increased communication latency arising from collective MPI calls. HOOMD-blue and LAMMPS-GPU scaling are in otherwise good qualitative agreement, indicating the absence of major bottlenecks in either code. HOOMD-blue performance is superior to that of LAMMPS-GPU by roughly a factor of two, which impressively demonstrates the speed-up gained by optimizing for the GPU exclusively and highlights some present limitations of a hybrid GPU/CPU approach. The hybrid approach used by LAMMPS relies on offloading only the compute-intensive force computation to the GPU and on using the GPU in a sparse fashion. In turn, for maximum performance GPU kernel launches from several MPI ranks per node are multiplexed onto the same GPU (using the HyperQ capability of NVIDIA Kepler GPUs), which can create additional kernel launch overhead. For HOOMD-blue, we varied the number of CPU cores assigned to a GPU and did not find any benefit from using more than one core per GPU.

\subsection{Strong scaling}

We measured HOOMD-blue performance for the strong scaling of a simple LJ liquid benchmark of $N=10,976,000$ particles (for the benchmark script, see [48]) on Titan. We compared HOOMD-blue performance to the performance of 


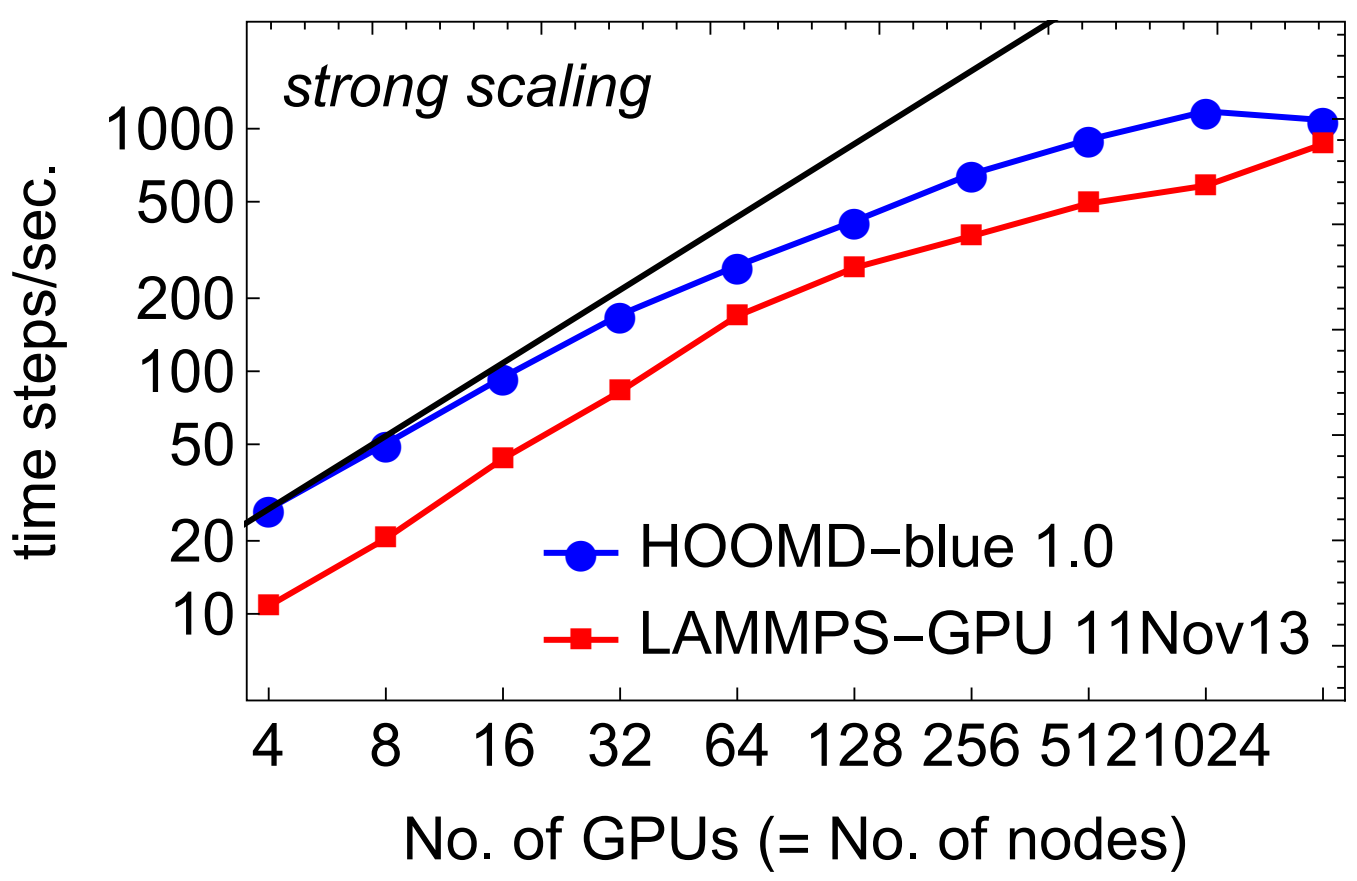

Figure 7: (color online) Strong scaling of a LJ liquid benchmark with $N=10,976,000$ particles (single precision) on the Titan supercomputer (Cray XK7, Oak Ridge National Laboratories). Shown is the performance in terms of number of time steps per second (TPS) for HOOMD-blue (circles) and LAMMPS-GPU (squares), running on $P=4 \ldots 2048$ GPUs. HOOMD-blue performance is optimal with 1 MPI rank per node/GPU, for LAMMPS-GPU up to $16 \mathrm{CPU}$ cores are assigned to one device. The solid line shows ideal linear scaling.

the LAMMPS-GPU package, and the details of the simulation are otherwise the same as for the weak scaling.

Figure 7 shows HOOMD-blue performance, i.e. number of time steps per second vs. number of GPUs, compared to LAMMPS-GPU for the LJ liquid benchmark, on up to 2,048 GPUs. As can be seen, essentially ideal scaling holds up to 32 GPUs, beyond which the scaling shows decreased efficiency. However, scaling holds up to 1,024 GPUs in this benchmark. Both HOOMD-blue 


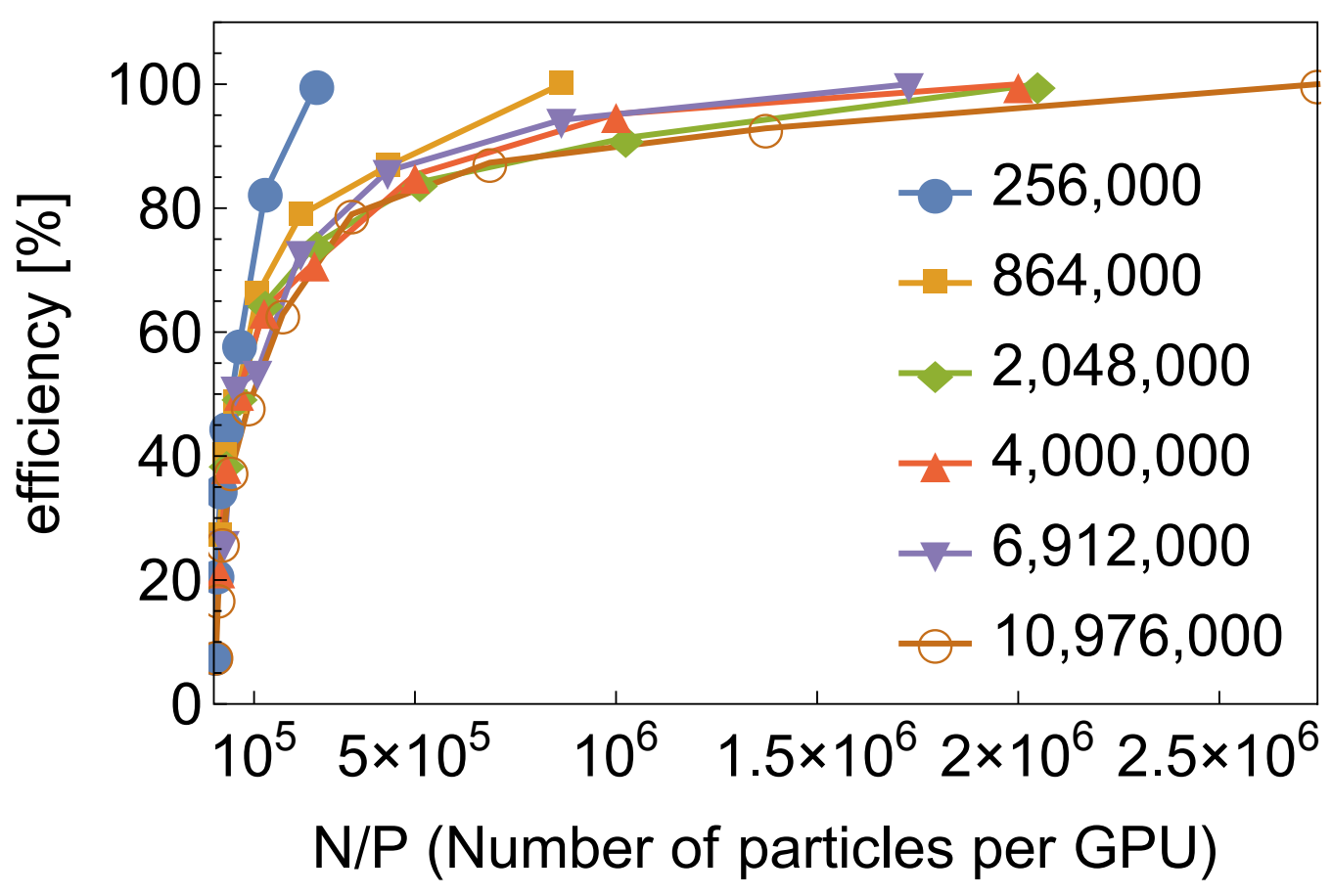

Figure 8: (color online) Strong scaling efficiency of HOOMD-blue in a LJ liquid benchmark for seven different total system sizes between 256,000 and 10,976,000 particles (single precision), running on at least $P_{\min }$ up to 2048 GPUs (for the largest system size) of Titan. Shown is the normalized performance TPS $(P) P_{\min } / P \operatorname{TPS}\left(P=P_{\min }\right)$ vs. number of particles per GPU $N / P$.

and LAMMPS-GPU scale qualitatively similarly, such that they are limited by architecture-inherent communication bottlenecks in the same way. However, as for weak scaling (Sec. 6.1), HOOMD-blue achieves a roughly constant, two-fold speed-up over the LAMMPS-GPU code, demonstrating the effectiveness of the above optimizations.

\subsection{Scaling efficiency}

Only in rare circumstances, however, does the maximum achievable performance matter; more often the compute resources are limited and shared with 
other researchers. It is therefore relevant to know what is the maximum number of GPUs a simulation can be run with to reach a prescribed minimum scaling efficiency. To this end, we compared strong scaling efficiency at various total system sizes, for the same system as studied in Sec. 6.2. The result is shown in Fig. 8 for six different total system sizes between $N=256,000$ and $N=10,976,000$ particles, on different numbers of GPUs up to 2,048 GPUs. It turns out that the strong scaling performance is essentially independent of the total size. With the slight exception of the $P=1$ and $P=2$ data points for the smallest system $(N=256,000)$, for which we expect the largest relative amount of communication overheads, the data falls onto a master curve obtained from plotting all results as scaling efficiency $\gamma=\operatorname{TPS}(P) / P \operatorname{TPS}(P=1)$ vs. number of particles $N / P$ per GPU. In cases where at least $P_{\min }>1$ GPUs are required to hold the system in memory, we normalize by $\operatorname{TPS}\left(P_{\min }\right) / P_{\min }$.

The data in Fig. 8 shows that maximum scaling efficiency $(\gamma \sim 1)$ is achieved for $N / P \gtrsim 10^{6}$. However, a very good efficiency of $70 \%$ is already achieved with $N / P \sim 200,000$ particles per GPU, which is easily reached by many medium- to large scale simulations e.g. of polymeric liquids.

\section{Polymer brush scaling benchmark}

We also analyze the scaling performance of a more complex benchmark system, a polymer brush in presence of a polymeric solvent. Chemical details are described at the level of a united atom model [49]. The solvent is hexane, and the tethers are oleic acid molecules, grafted onto an immobile wall. The system is used to study effective interactions of polymer-tethered surfaces [50]. Here we use it as a model system to study HOOMD-blue performance under conditions 


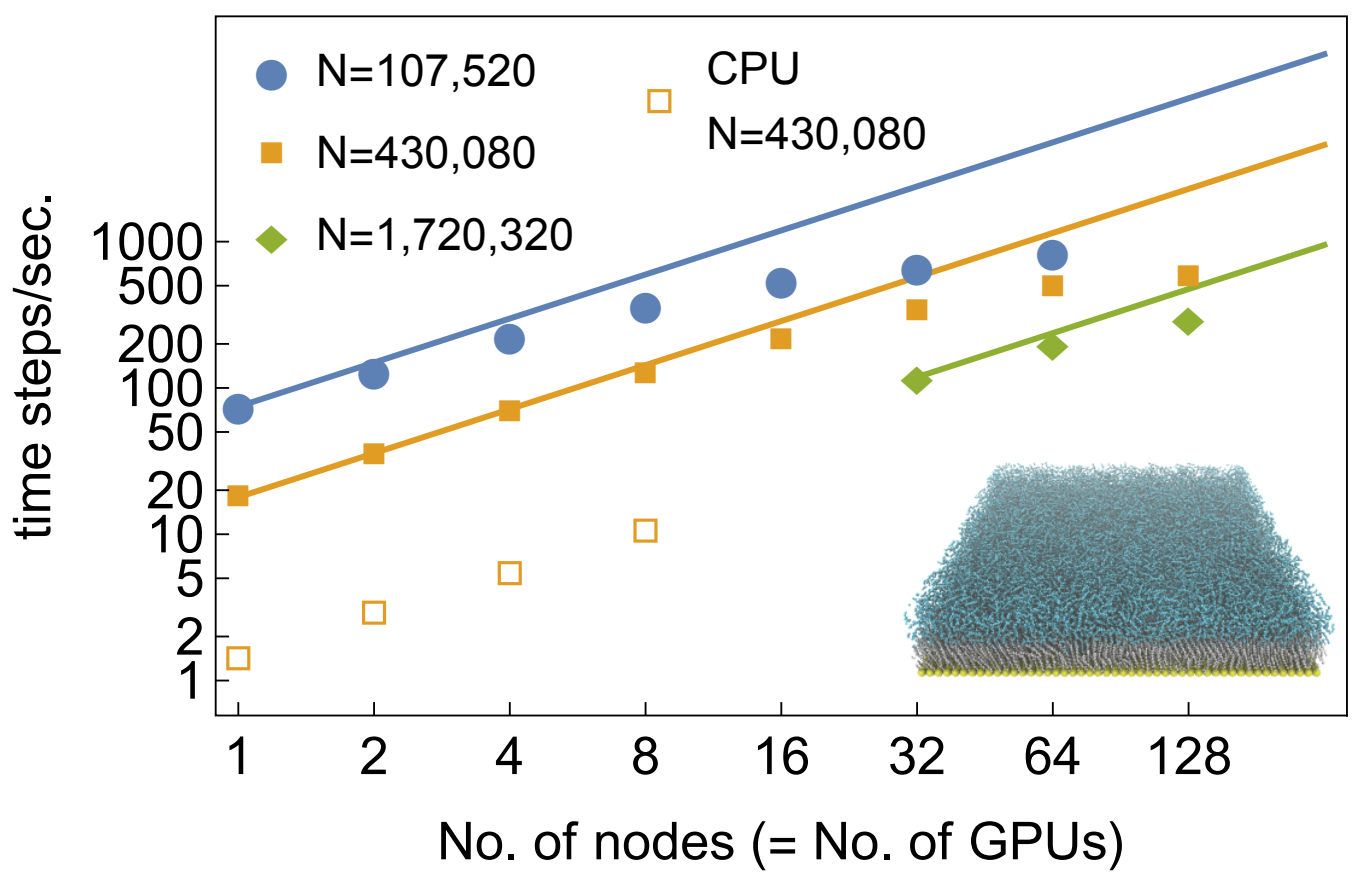

Figure 9: (color online) Benchmark for an oleic acid brush in hexane solvent. Shown is the performance in terms of number of time steps per second vs. number of GPUs or number of CPU sockets for three different total system sizes. Solid symbols: GPU benchmark at different total system sizes, open symbols: CPU benchmark at $\mathrm{N}=430,080$. Because of memory limitations, the smallest number of GPUs for the system of $1,720,320$ particles is 32 , and because of the minimum size required for the subdomain in a spatial domain decomposition, the largest number of nodes in the CPU benchmark is eight. Solid lines show ideal linear scaling. 
that are more computationally demanding than a LJ liquid.

The polymer backbones are parameterized using bond, angle and dihedral potentials, and the system is equilibrated at constant pressure and temperature (NPT ensemble). For this bennchmark, we replicate the initial configuration along the wall (or $\mathrm{x}-\mathrm{y}$ ) dimension using system.replicate(), to produce three different total system sizes (numbers of polymeric particles) of $N=107,520,430,080$ and $1,720,320$. Detailed parameters of the benchmark, which was run on Blue Waters, are documented in the supplementary information [48]. As shown in Fig. 9, the performance (time steps / second) scales approximately linearly with the number of GPUs, up to a maximum number of GPUs that increases with total system size. For example, for the system of 430,080 polymer beads, saturation sets in at 32 GPUs, corresponding to 13,440 particles per GPU. For this system, very good scaling is observed and a maximum speed-up of a factor $\approx 32$ over single-GPU execution is achieved, which shows that the speed-ups obtained in the LJ benchmarks are robust and transferable to more complex interaction models.

Moreover, we compare the GPU to CPU performance of HOOMD-blue running on the $16 \mathrm{CPU}$ cores of Blue Waters' XK7 nodes (1 AMD 6276 Interlagos CPU, 16 integer scheduling units, nominal clock speed $2.3 \mathrm{GHz}$ ), for the $N=430,080$ benchmark. The strong scaling data for time steps per second vs. number of nodes is shown in Fig. 9, open symbols, and demonstrates an average speed-up of a factor of TPS $(n$ GPUs $) /$ TPS $(n$ CPUs $) \approx 12.5$. 

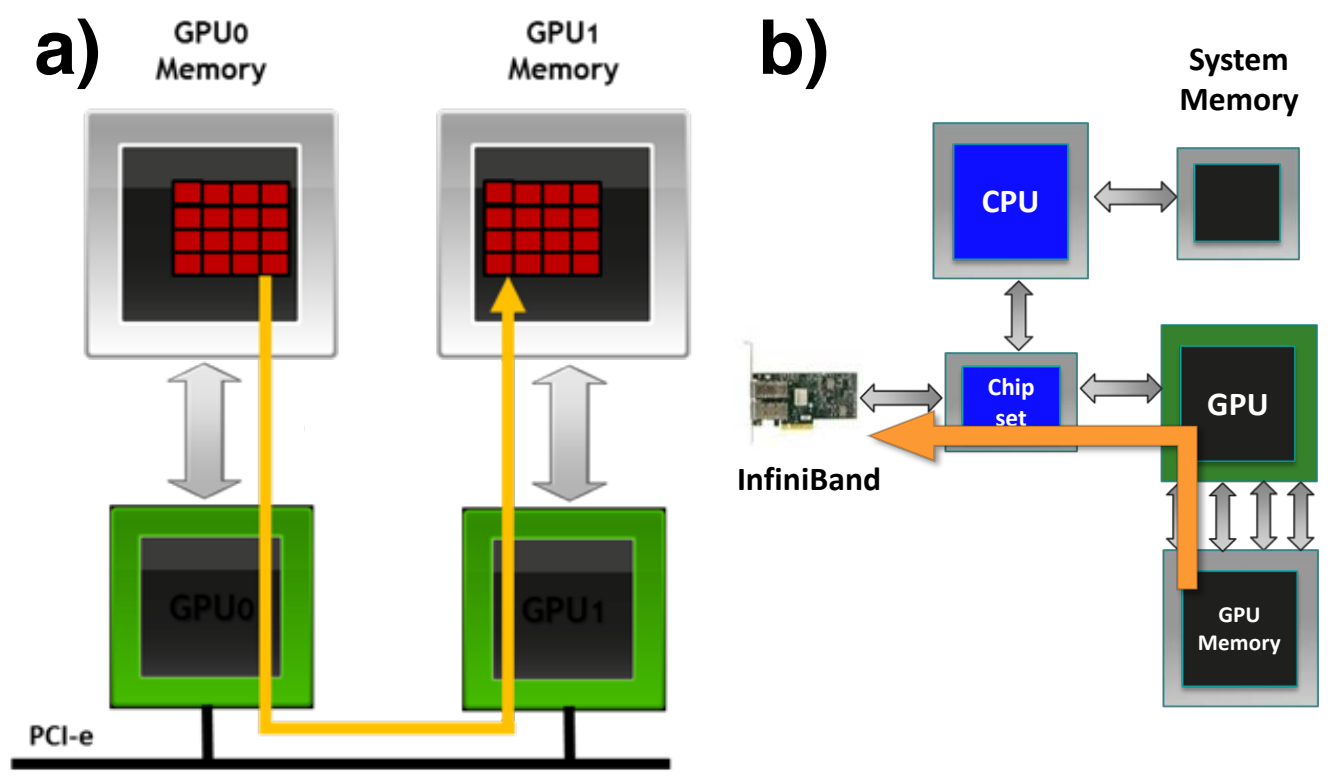

Figure 10: (color online) RDMA enabled technologies for data transfer between GPUs across the PCIe bus without involving the CPU. a) Intra-node Peer-to-peer b) GPUDirect RDMA. Reproduced from the GPUDirect webpage [51]. 


\section{Strong scaling with GPUDirect RDMA}

\subsection{Technological background}

Since rapid developments in GPU hardware have out-paced the speeds at which system architecture, and particularly PCIe, can transfer data (16 GB/s peak bandwidth in PCIe generation 3), this has generated pressure on the development of latency-optimized technologies for GPU-to-GPU communication, either over a PCIe switch, with two GPUs connected to the same switch, or via the intermediate of a network interface card (NIC) for Infiniband connected to the same PCIe segment. The first generation implementation of a hardware-accelerated communication path for GPUs was GPUDirect v1 [52], which eliminates unnecessary buffer copies within host memory for staging data to the NIC. To use GPUDirect, it is essentially only required that the send/receive buffers are page-locked, which enables fast access by the network card. However, with GPUDirect v1, the data still needs to be available in host memory, making it necessary to copy the data to and from there for sending and receiving.

With the release of GPUDirect v1, MPI stacks have been developed (starting with MVAPICH2 1.8 and OpenMPI 1.7, and others) that accept memory pointers for the staging buffers, designed to relieve the application developer from having to manually optimize staging protocols and or pipelining techniques performance $[53,54,20]$.

The second version of the GPUDirect technology, introduced with Fermi generation GPUs, offers the possibility of intra-node GPU-GPU transfer using the RDMA feature of PCIe, without involving the CPU (Fig. 10, left panel). Using this technique, up to $80-90 \%$ of PCIe peak bandwidth can be achieved for these transfers. However, because peer-to-peer transfers are limited to a single node, 
transfers that involve inter-node communication e.g. over Infiband are not optimized.

The latest generation of the GPUDirect technology, GPUDirect RDMA (GDR), solves this problem by extending RDMA access to the NIC and allows fast data transfer between remote GPUs, completely offloading the CPU from the communication burden (Fig. 10, right panel). All of these protocols are supported in the MPI libraries MVAPICH2 2.0 GDR, and in OpenMPI 1.7.4 and later. In the following, we analyze the efficiency and discuss limitations of this advanced technology in the example of HOOMD-blue scaling benchmarks.

\subsection{GPUDirect RDMA benchmarks}

The Wilkes cluster at the University of Cambridge is a large GPU cluster designed to fully leverage GPUDirect RDMA. The system features 128 nodes with two NVIDIA Tesla K20c GPUs for each node, dual-rail Mellanox Connect-IB Infiniband and NVIDIA GPUDirect RDMA software stack. The cluster is mainly being used for academic research. We assess the performance of HOOMD-blue on this system by running the standard LJ benchmark that comes with HOOMD-blue, with a total system size of $N=64,000$ particles $\left(\mathrm{NVT}, \delta t=0.005, r_{\text {buff }}=0.4\right.$, $\left.T=1.2, r_{\text {cut }}=3.0, \epsilon=\sigma=1.0\right)$. Fig. 11 shows the scaling behavior, both for single-precision (upper panel) and double-precision (lower panel) builds. As can be seen, in single precision the default version of HOOMD-blue, which does not take advantage of CUDA-aware MPI at all, always performs better than either CUDA-aware MPI or CUDA-aware MPI with GPUDirect RDMA. The size of message transferred in (32bit) single precision, which is half of that transferred in (64bit) double precision, is too small to take advantage of full communication bandwidth. 

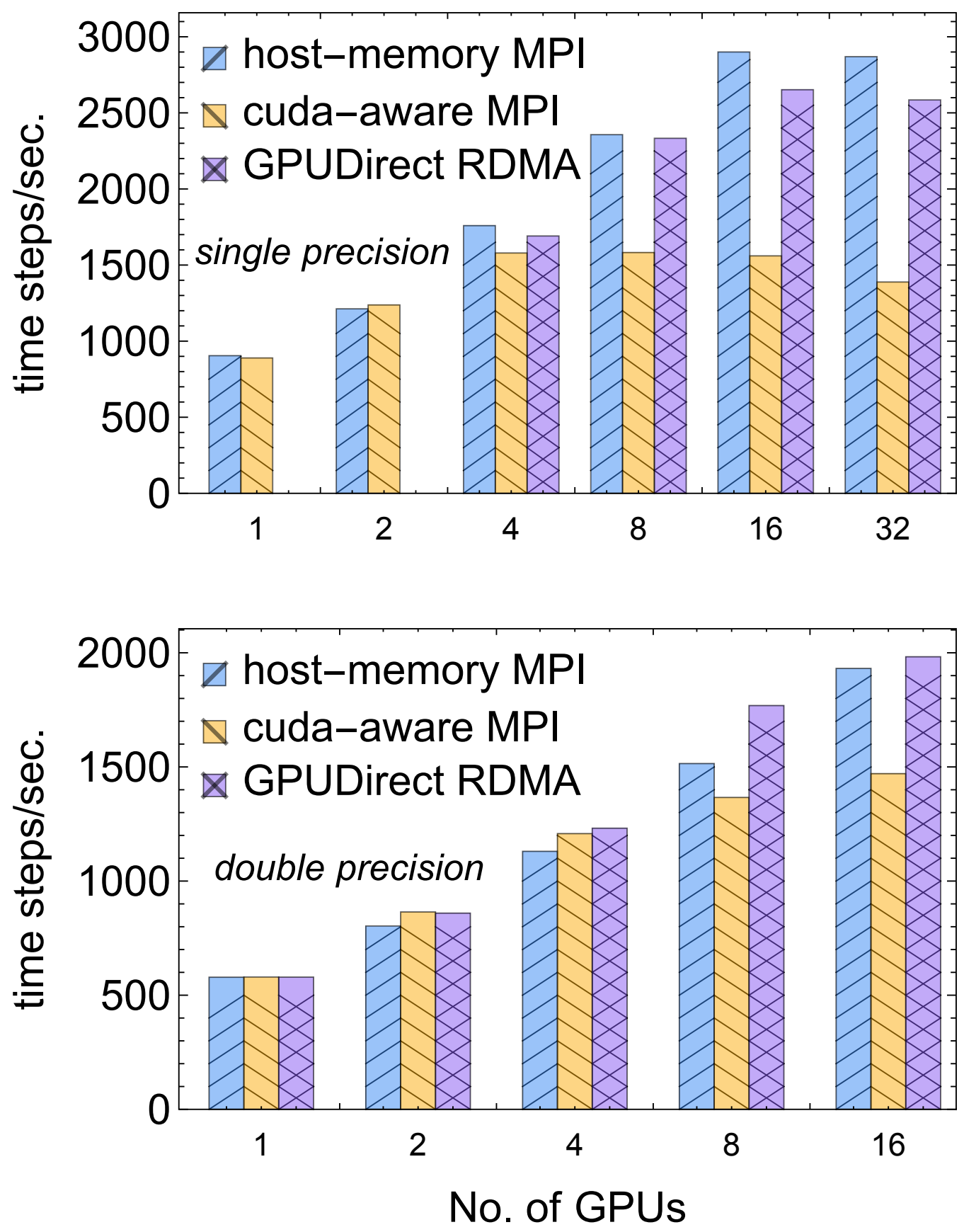

Figure 11: (color online) Strong scaling benchmark for a GPUDirect RDMA benchmark of $N=64,000$ particles LJ liquid benchmark on the Wilkes GPU cluster, comparing default hostmemory MPI, CUDA-aware MPI, and CUDA-aware MPI with GPUDirect RDMA (GDR) using MVAPICH 2.0 GDR (experimental). Shown is the performance in number of time steps per second vs. number of GPUs, for single precision (top) and double-precision (bottom) runs. 


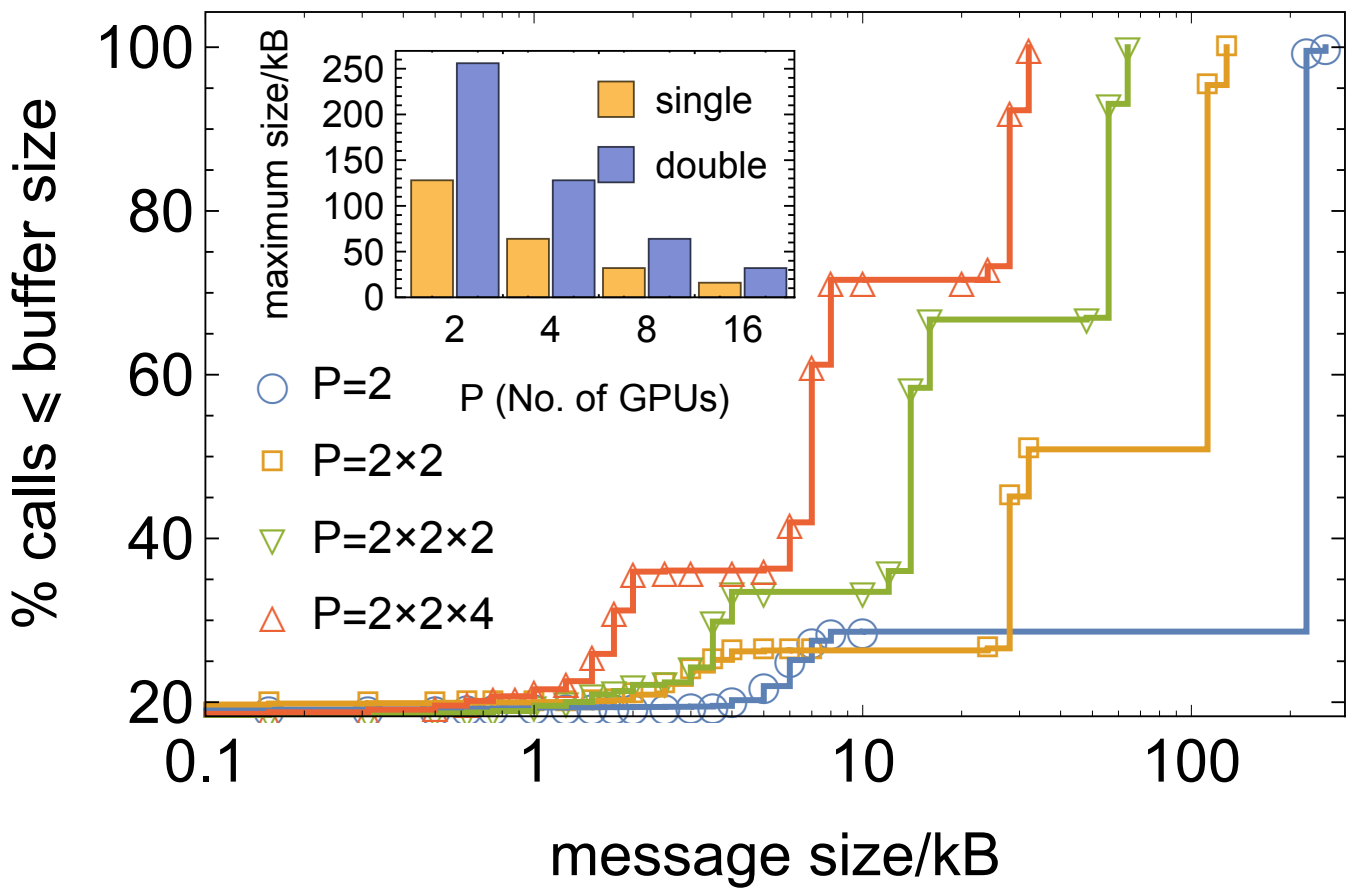

Figure 12: (color online) Distribution of message sizes for the benchmark of Fig. 11 (lower panel). Shown is the cumulative relative frequency of message sizes in neighbor communication (MPI_Isend/recv) in double-precision runs, for different numbers of GPUs, obtained with the IPM tool [55]. Inset: Maximum message size in $\mathrm{kB}$ as function of the number $P$ of GPUs, in single (light shaded/yellow) and double precision (dark shaded/blue).

On the other hand, for double-precision mode, the lower panel of Fig. 11 shows a performance benefit of enabling CUDA-aware MPI on up to 4 nodes, or $N / P=16,000$. More interestingly, we observe better performance of GPUDirect RDMA than both plain CUDA-aware MPI and host memory staging. This demonstrates the potential benefit of GPUDirect RDMA technology in strongscaling applications.

Figure 12 shows the cumulative frequency of the message size of MPI_Irecv calls during execution of the double-precision LJ benchmark, for which data is 
shown in Fig. 11 (bottom panel). The distribution has multiple 'knees', which are characteristic of the communication pattern described in Sec. 4.3.1. The precise location of these knees depends on the details of the domain decomposition, however the maximum message size affects performance through various internal thresholds of the MPI library. In the case of GPUDirect RDMA, we were able to use maximum optimal thresholds of $32 \mathrm{~KB}$, above which the MPI library switches to default pipelined communication. Interestingly, this limit is reached with at least eight GPUs in single precision, or 16 GPUs in double precision, for the $N=64,000 \mathrm{LJ}$ benchmark, as shown in Fig. 12, inset. We confirm that for these minimum numbers of GPUs the GPUDirect RDMA enabled benchmarks indeed perform superior to cuda-aware MPI (Fig. 11), however the effective performance is in the range of the optimized host memory implementation.

\subsection{Performance comparison to a CUDA-aware MPI enabled port of LAMMPS}

To assess whether HOOMD-blue makes optimal use of the CUDA-aware MPI based communication protocols, we compare against another port of LAMMPS on GPUs, LAMMPS-Kokkos, a recent alternative to LAMMPS-GPU. The Kokkos package inside LAMMPS is a forward looking capability with support for other accelerators (Intel Xeon Phi), but with very limited feature support at the moment. The package supersedes a previous port of LAMMPS on GPUs, LAMMPSCUDA [4]. It also offers support for CUDA-aware MPI implementations, which makes it interesting to compare to HOOMD-blue performance here.

As a benchmark system we choose the double precision LJ system benchmark supplied with the Kokkos package (NVE, $N=2,097,152, \delta t=0.005, r_{\text {buff }}=0.3$, $r_{\text {cut }}=2.5, \epsilon=\sigma=1.0$ ), with the only change that we increase the neighbor list build frequency to every six time steps, to ensure correct computation of forces. 


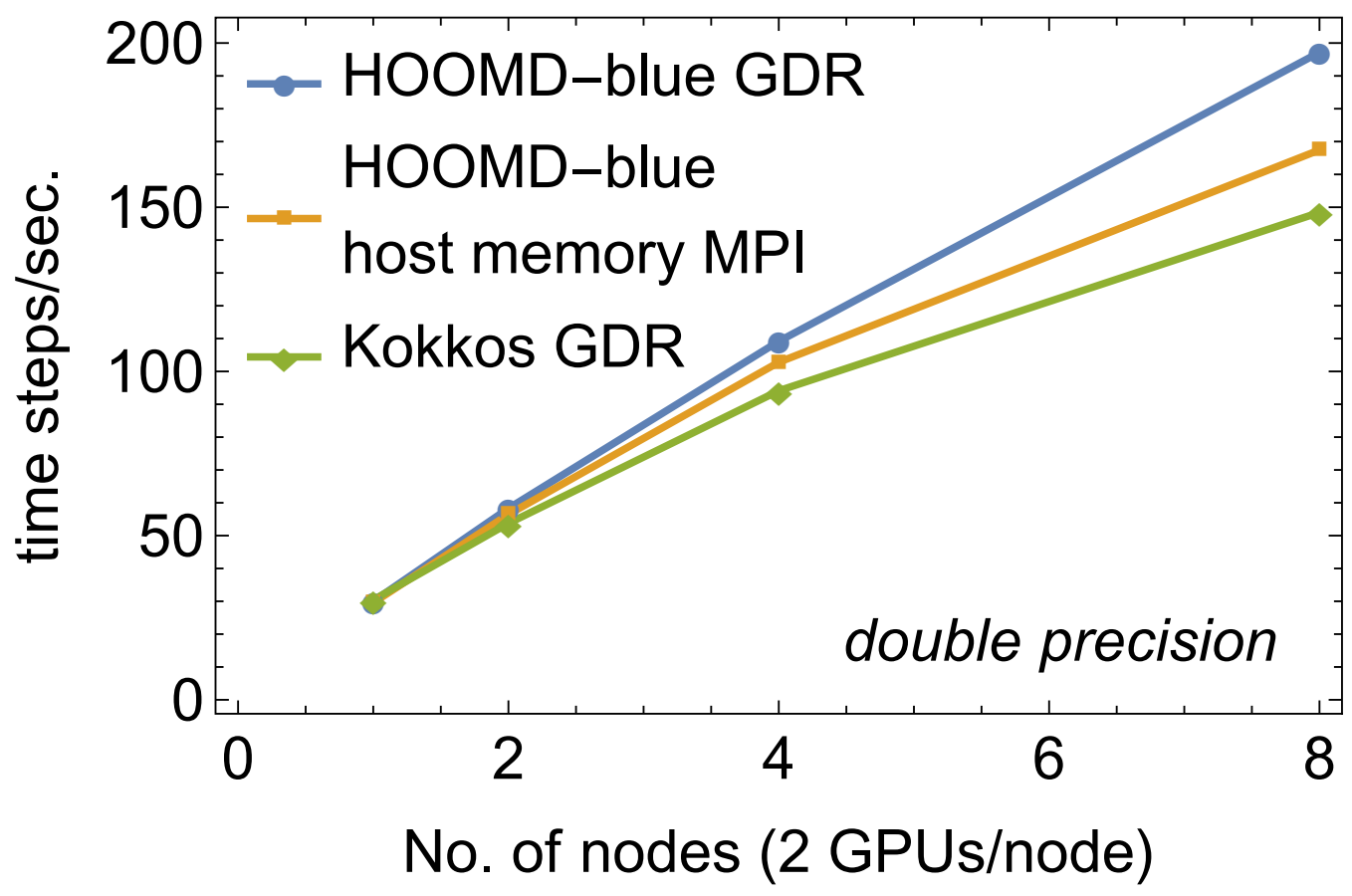

Figure 13: (color online) Strong scaling of a double-precision LJ liquid benchmark of $N=$ 2, 097, 152 particles comparing the performance of HOOMD-blue performance (with GDR or with host memory MPI) and of LAMMPS-Kokkos (with GDR), on $P=1 \ldots 8$ GPUs. Shown is performance in time steps per second vs. number of GPUs. 
The corresponding HOOMD-blue simulations start from the same fcc lattice initial configuration (thermalized at $T=1.44$ ), additionally equilibrated over 30,000 time steps. For the HOOMD-blue simulations we choose the optimal value of $r_{\text {buff }}$ and the distance check interval by prior tuning. Figure 13 shows the performance of the Kokkos package, where LAMMPS is run in device communication mode and with GPUDirect RDMA enabled, and for different build and runtime settings of HOOMD-blue. The agreement of single-GPU performance emphasizes that both Kokkos and HOOMD-blue are essentially fully optimized for simulations at this particle number, where they are limited by device memory bandwidth. On the other hand, on eight nodes the GDR version of HOOMD-blue performs better than the Kokkos package by a factor of about 1.4, which we attribute to the optimizations of the communication algorithm described in Sec. 4.3.

\section{Strong scaling of a DPD benchmark}

We also compare dissipative particle dynamics (DPD) performance between HOOMD-blue and two other codes. The communication pattern differs from that of $\mathrm{LJ}$ in two ways. Velocities of ghost particles need to be communicated, in order to compute the drag term in the DPD force. Hence, twice the amount of data is communicated per time step. Moreover, to correctly seed per-particle-pair random number generators [41], global particle IDs of ghost particles additionally need to be communicated with every ghost exchange. Recently, Tang and Karniadakis [6] presented a GPU-optimized implementation of DPD, validated and benchmarked on the Titan supercomputer. They demonstrate excellent strong scaling properties. The software is available within LAMMPS as the USER-MESO package [56].

Here, we compare HOOMD-blue performance on the Cray XK7 to the bench- 


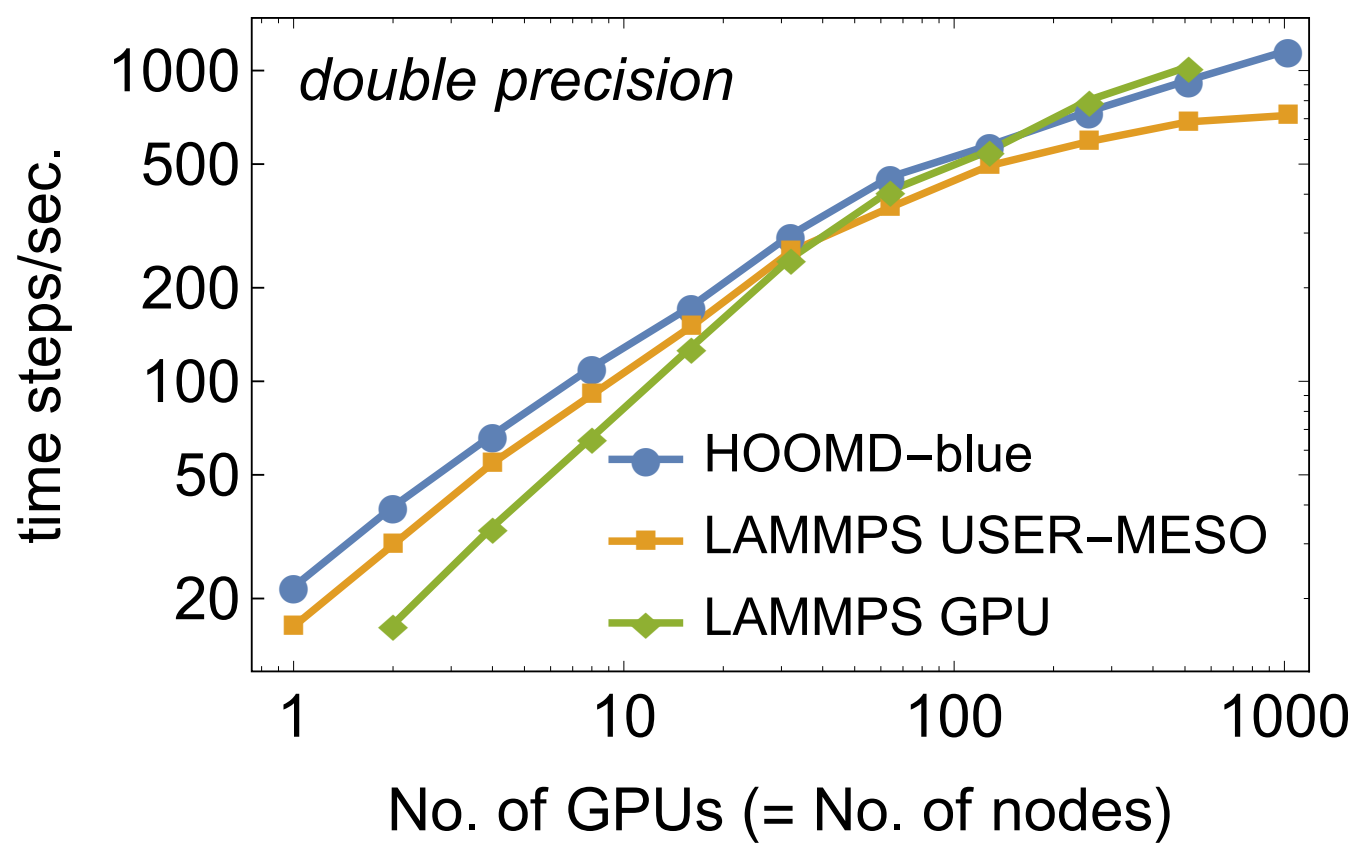

Figure 14: (color online) Strong scaling performance of a DPD benchmark of $N=2,000,000$ particles, comparing HOOMD-blue, LAMMPS USER-MESO and LAMMPS-GPU. Shown is the number of time steps per second, vs. number of GPUs, for $P=1 \ldots 1,024$ GPUs. 
mark numbers reported in Ref. [6] (Fig. 14 therein), for the same benchmark of $N=2,000,000$ particles. We also compare to LAMMPS-GPU performance [57]. The simulation details are: $\delta t=0.005$ at number density $\rho=3$, and with DPD parameters $A=25, \gamma=4.5$. HOOMD-blue and LAMMPS-GPU use full double precision, the USER-MESO package is run in mixed precision: communication is performed in double precision, but node local force evaluations are performed in single precision. As in the previous benchmarks, HOOMD-blue performance was optimized by tuning the value $r_{\text {buff }}$ and the distance check period. The number of time steps per second was measured after an additional warm-up of 30,000 time steps, averaged over 50,000 steps. LAMMPS-GPU and USER-MESO benchmarks were performed on Titan, HOOMD-blue benchmarks were performed on the Blue Waters machine at the National Center for Supercomputing Applications. Even though this machine has the same architecture as Titan (Cray XK7), we expect slight differences in performance, from differences in system software and network configuration. Figure 14 shows the number of time steps per second as a function of the number of GPUs in strong scaling, on up to 1,024 GPUs. Performance values are maxima from several runs (up to $10 \%$ variability was observed for large runs). Remarkably, the performance of HOOMD-blue parallels that of the USER-MESO package over the whole range of numbers of GPUs and appears to be only slightly superior (15\%), but outperforms LAMMPS-GPU for small $(\lesssim 32)$ numbers of GPUs. However, for larger numbers of nodes the performance between the codes is comparable, which we attribute to the difference in communication patterns of DPD vs. LJ, where for DPD in double precision, an amount of data four times larger is communicated than for $\mathrm{LJ}$ in single precision. The DPD benchmark is therefore less sensitive to the latency optimizations we 
focus on in this contribution, and given that it is likely bandwidth-bound, further underscores that with GPUs the communication bandwidth of current system architectures, along with various sources of latency, has become the main limiting factor of MD performance.

\section{Conclusion and Outlook}

We gave a detailed account of how we ported HOOMD-blue to a distributed memory model (MPI). Because HOOMD-blue is a fully GPU-enabled code, a particular challenge was presented by the latency of device-to-device communication. We addressed this challenge using a highly optimized communication algorithm. Our communication routines are implemented on the GPU to reduce the amount of data transferred over PCIe and allow us to take advantage of CUDA-aware MPI libraries. We also optimized for strong scaling on thousands of GPUs, which we achieved using a design for the neighbor list and force computation kernels based on cooperative thread arrays and an auto-tuning algorithm.

We evaluate the performance of our code in terms of both weak and strong scaling benchmarks, for which we compared it to similarly optimized implementations of GPU-enabled MD, and find HOOMD-blue performance to be equivalent or superior. HOOMD-blue exhibits qualitatively similar scaling behavior to these other codes, indicating that our optimizations are successful, and that the scaling limits inherent to the underlying architecture have been reached. We note that the GPU-centric design of HOOMD-blue is different from other more traditional MD codes, which have started as CPU-only codes.

In the case of GPUDirect RDMA, we find superior performance in double precision benchmarks, demonstrating the usefulness of the technology, especially in 
strong scaling situations, however also its current limitations. To further improve strong scaling performance, latency and bandwidth bottlenecks will have to be reduced. Moreover, a closer integration of the GPU into the communication path seems realistic, such as to provide the capability of GPU kernel call-backs from MPI calls. In general, we anticipate that future designs will tightly couple GPUs as throghput-optimized and CPUs as latency-optimized compute components, and optimal code performance will depend on high-bandwidth links between the processors [58], to achieve greater concurrency.

In this first 1.0 release of HOOMD-blue with MPI, we did not enable multiGPU support for electrostatics calculations, rigid bodies or anisotropic particles, available only in single-GPU simulations. We expect to implement these capabilities in future versions. The current implementation exclusively relies on spatial domain decomposition as a work distribution technique and thus applies to mostly homogeneous systems, whereas sophisticated load-balancing schemes have been implemented for more inhomogoeneous or biomolecular systems [59, 60, 31] on CPUs, and they should additionally benefit from GPU acceleration.

More broadly, our study further establishes GPUs as extremely fast engines for MD simulation compared to traditional CPU cores. GPUs not only realize an order of magnitude speed-up over current-generation CPUs, but they also scale very well using spatial domain decomposition. Hence, our and comparable codes therefore both greatly benefit from the unprecedented performance offered by these fast processors, and at the same time they push the envelope of current system designs. Since the speed-ups presented here rely chiefly on exploiting parallelism at various levels, i.e. by using GPUs on the node-level, and by scaling the code up to many nodes, we provide a very clear case for how parallelism can be 
the main enabling strategy in computational physics discovery.

Acknowledgments. We gratefully acknowledge helpful discussions with Davide Rosetti, Yu-Hang Tang, Dhabaleswar K. Panda, and Christian Trott. We thank Rong Shi for providing benchmark data for GPUDirect RDMA in single precision.

This material is based upon work supported by the DOD/ASD (R\&E) under Award No. N00244-09-1-0062 (JG, JAA, JAM, SCG). JG acknowledges support by DFG grant GL733/1-1. We also acknowledge support by the National Science Foundation, Division of Materials Research, award DMR 1409620 (JAA and SCG), and award DMR 0907338 (JG and DCM). This work was partially supported by a Simons Investigator award from the Simons Foundation to Sharon Glotzer (SCG, JG). This research used resources of the Oak Ridge Leadership Computing Facility at the Oak Ridge National Laboratory, which is supported by the Office of Science of the U.S. Department of Energy under Contract No. DEAC05-00OR22725. This research is part of the Blue Waters sustained-petascale computing project, which is supported by the National Science Foundation (award number ACI 1238993) and the state of Illinois. Blue Waters is a joint effort of the University of Illinois at Urbana-Champaign and its National Center for Supercomputing Applications. We thank the University of Cambridge for providing access to their Wilkes cluster. Any opinions, findings, and conclusions or recommendations expressed in this publication are those of the author(s) and do not necessarily reflect the views of the DOD/ASD(R\&E). The Glotzer Group at the University of Michigan is a CUDA Research Center. Hardware support by NVIDIA is gratefully acknowledged.

Author contributions. JG and JAA implemented the algorithm. TDN performed benchmarks on Titan, and JG performed benchmarks on Blue Waters. PL and FS 
performed benchmarks on Wilkes. JM provided the polymer brush benchmark. DCM advised research at the University of Minnesota and provided ideas for the implementation of the bond communication algorithm, and SCG advised research at the University of Michigan. All authors contributed to the manuscript.

[1] Anderson, J. A., Lorenz, C. D., and Travesset, A., Journal of Computational Physics 227 (2008) 5342.

[2] Grand, S. L., Götz, A. W., and Walker, R. C., Computer Physics Communications (2012).

[3] Plimpton, S., J. Comp. Phys. 117 (1995) 1.

[4] Trott, C. R., LAMMPSCUDA - a new GPU accelerated Molecular Dynamics Simulations Package and its Application to Ion-Conducting Glasses, $\mathrm{PhD}$ thesis, 2011.

[5] Brown, W. M., Wang, P., Plimpton, S. J., and Tharrington, A. N., Computer Physics Communications 182 (2011) 898.

[6] Tang, Y.-H. and Karniadakis, G. E., Computer Physics Communications 185 (2014) 2809.

[7] Páll, S. and Hess, B., Computer Physics Communications 184 (2013) 2641.

[8] Stone, J. E. et al., Journal of Computational Chemistry 28 (2007) 2618.

[9] Phillips, J., Stone, J., and Schulten, K., High Performance Computing, Networking, Storage and Analysis, 2008. SC 2008. (2008) 1. 
[10] Eastman, P. et al., Journal of Chemical Theory and Computation 9 (2013) 461.

[11] Lysaght, M., Uchronski, Mariusz Kwiecien, Agnieszka Gebarowski, Marcin Nash, P., Girottoa, I., and Todorovc, I. T., PRACE whitepaper, PRACE-1IP

[12] Harvey, M. J., Giupponi, G., and Fabritiis, G. D., Journal of Chemical Theory and Computation 5 (2009) 1632.

[13] Deng, H., Li, X., Liu, X., and Wang, G., 2011 40th International Conference on Parallel Processing Workshops (2011) 191.

[14] Roehm, D. and Arnold, A., The European Physical Journal Special Topics 210 (2012) 89.

[15] Ganesan, N., Taufer, M., Bauer, B., and Patel, S., 2011 IEEE International Symposium on Parallel and Distributed Processing Workshops and Phd Forum (2011) 472.

[16] Colberg, P. and Höfling, F., Computer Physics Communications 182 (2011) 1120.

[17] HOOMD-blue, http://codeblue.umich.edu/hoomd-blue.

[18] Carter Edwards, H., Trott, C. R., and Sunderland, D., Journal of Parallel and Distributed Computing (2014).

[19] GPUDirect RDMA, http://docs.nvidia.com/cuda/gpudirect-rdma/. 
[20] Potluri, S., Hamidouche, K., Venkatesh, A., Bureddy, D., and Panda, D. K., 2013 42nd International Conference on Parallel Processing (2013) 80.

[21] HOOMD-blue homepage https://codeblue.umich.edu/hoomdblue/publications.html.

[22] Glaser, J., Qin, J., Medapuram, P., and Morse, D. C., Macromolecules (2014) 140113062817000 .

[23] Glaser, J., Medapuram, P., Beardsley, T. M., Matsen, M. W., and Morse, D. C., Phys. Rev. Lett. 113 (2014) 068302.

[24] Reith, D., Mirny, L., and Virnau, P., Progress of Theoretical Physics Supplement 191 (2011) 135.

[25] Levine, B. G. et al., Journal of Chemical Theory and Computation 7 (2011) 4135 .

[26] Reith, D., Milchev, A., Virnau, P., and Binder, K., EPL (Europhysics Letters) 95 (2011) 28003.

[27] Lin, B., Martin, T. B., and Jayaraman, A., ACS Macro Letters 3 (2014) 628.

[28] Nguyen, T. D., Carrillo, J.-M. Y., Matheson, M. a., and Brown, W. M., Nanoscale 6 (2014) 3083.

[29] Nguyen, T. D., Fuentes-Cabrera, M., Fowlkes, J. D., and Rack, P. D., Physical Review E 89 (2014) 032403.

[30] Lam, C.-H. and Tsui, O. K. C., Physical Review E 88 (2013) 042604. 
[31] Grime, J. and Voth, G., Journal of Chemical Theory and Computation 10 (2014) 423.

[32] Phillips, C. L., Iacovella, C. R., and Glotzer, S. C., Soft Matter 6 (2010) 1693.

[33] Wilms, D., Virnau, P., Sengupta, S., and Binder, K., Physical Review E 85 (2012) 061406.

[34] Zhang, G., Stillinger, F. H., and Torquato, S., Physical Review E 88 (2013) 042309 .

[35] Nguyen, N. H. P., Jankowski, E., and Glotzer, S. C., Physical Review E 86 (2012) 011136.

[36] Perlmutter, J. D., Qiao, C., and Hagan, M. F., eLife 2 (2013) e00632.

[37] Benedetti, F., Dorier, J., and Stasiak, A., Nucleic Acids Research 42 (2014) 10425.

[38] Kapoor, A. and Travesset, A., Proteins 81 (2013) 1200.

[39] Martyna, G. J., Tobias, D. J., and Klein, M. L., J. Chem. Phys. 101 (1994) 4177.

[40] Martyna, G. J., Tuckerman, M. E., Tobias, D. J., and Klein, M. L., Molecular Physics 87 (1996) 1117.

[41] Phillips, C. L., Anderson, J. A., and Glotzer, S. C., Journal of Computational Physics 230 (2011) 7191.

[42] Anderson, J. A. and Glotzer, S. C., arxiv 1308.5587 (2013). 
[43] CUDA-aware MPI (nVidia), http://devblogs.nvidia.com/parallelforall/introductioncuda-aware-mpi/.

[44] Baxter, S., ModernGPU, http://nvlabs.github.io/moderngpu/.

[45] Bell, N. and Hoberock, J., Thrust: A Productivity-Oriented Library for CUDA, in GPU Computing Gems, Jade Edition, edited by Hwu, W.-m. W., chapter 26, pages 359-373, Morgan Kaufmann, 2012.

[46] Brown, W. M., Kohlmeyer, A., Plimpton, S. J., and Tharrington, A. N., Comp. Phys. Comm. 183 (2012) 449.

[47] CUDA Programming Guide, http://docs.nvidia.com/cuda/cuda-cprogramming-guide.

[48] Supplementary Information, at ...

[49] Paul, W., Yoon, D. Y., and Smith, G. D., The Journal of Chemical Physics 103 (1995) 1702.

[50] Millan, J., In preparation.

[51] GPUDirect Webpage, http://developer.nvidia.com/gpudirect.

[52] Shainer, G. et al., Computer Science - Research and Development 26 (2011) 267.

[53] Wang, H. et al., Computer Science - Research and Development 26 (2011) 257.

[54] Wang, H., Potluri, S., Bureddy, D., Rosales, C., and Panda, D. K., IEEE Transactions on Parallel and Distributed Systems 25 (2014) 2595. 
[55] Skinner, D., Performance monitoring of parallel scientific applications, Technical report, Lawrence Berkeley National Laboratory (LBNL), Berkeley, CA, 2005.

[56] LAMMPS USER-MESO, http://www.cfm.brown.edu/repo/release/USER$\mathrm{MESO} /$.

[57] Nguyen, T. D. and Plimpton, S. J., Computational Materials Science in press (2014).

[58] NVLINK White paper, http://www.nvidia.com/object/nvlink.html.

[59] Phillips, J. C. et al., Journal of computational chemistry 26 (2005) 1781.

[60] Hess, B. and Kutzner, C., Journal of Chemical Theory and Computation 4 (2008) 435. 